\title{
Phytoalexin Accumulation in the Interaction Between Rice and the Blast Fungus
}

\author{
Morifumi Hasegawa, ${ }^{1}$ Ichiro Mitsuhara, ${ }^{2}$ Shigemi Seo, ${ }^{2}$ Takuya Imai, ${ }^{1}$ Jinichiro Koga, ${ }^{3}$ Kazunori Okada, ${ }^{4}$ \\ Hisakazu Yamane, ${ }^{4}$ and Yuko Ohashi ${ }^{2}$ \\ ${ }^{1}$ College of Agriculture, Ibaraki University, 3-21-1 Chuo, Ami, Ibaraki 300-0393, Japan; ${ }^{2}$ National Institute of Agrobiological \\ Sciences (NIAS), Kannon-dai 2-1-2, Tsukuba, Ibaraki 305-8602, Japan; ${ }^{3}$ Food and Health R\&D Laboratories, Meiji Seika \\ Kaisha, Ltd., Chiyoda Sakado 350-0289, Japan; ${ }^{4}$ Biotechnology Research Center, The University of Tokyo, 1-1-1 Yayoi, \\ Bunkyo-ku, Tokyo 113-8657, Japan
}

Submitted 24 December 2009. Accepted 9 April 2010.

\begin{abstract}
Blast fungus-induced accumulations of major rice diterpene phytoalexins (PA), momilactones $A$ and $B$, and phytocassanes A through $E$ were studied, focusing on their biosynthesis and detoxification. In resistant rice, all PA started to accumulate at 2 days postinoculation (dpi), at which hypersensitive reaction (HR)-specific small lesions became visible and increased 500- to 1,000-fold at $4 \mathrm{dpi}$, while the accumulation was delayed and several times lower in susceptible rice. Expression of PA biosynthetic genes was transiently induced at 2 dpi only in resistant plants, while it was highly induced in both plants at 4 dpi. Fungal growth was severely suppressed in resistant plants by 2 dpi but considerably increased at 3 to $4 \mathrm{dpi}$ in susceptible plants. Momilactone A treatment suppressed fungal growth in planta and in vitro, and the fungus detoxified the $P A$ in vitro. These results indicate that HR-associated rapid PA biosynthesis induces severe restriction of fungus, allowing higher PA accumulation in resistant rice, while in susceptible rice, failure of PA accumulation at the early infection stage allows fungal growth. Detoxification of $\mathrm{PA}$ would be a tactic of fungus to invade the host plant, and prompt induction of PA biosynthesis upon HR would be a trait of resistant rice to restrict blast fungus.
\end{abstract}

Hypersensitive reaction or response (HR) is the programmed cell death of infected plant cells to restrict pathogen invasion to the initially infected regions and is included in the resistance $(R)$ gene-mediated resistance to pathogens. Upon HR, the biosynthesis of low molecular-weight defense signal compounds, such as salicylic acid, jasmonic acid, and ethylene (De Laat et al. 1983; Iwai et al. 2006; Malamy et al. 1990; Seo et al. 2001), is induced for the signal transductions of disease resistance. As well as these signal compounds, antifungal molecules called phytoalexins (PA), which are also low molecular-weight compounds produced in host plants in response to parasite infection (Müller and Börger 1940) or stress (Kuć 1995), are induced in many plant-microbe interactions. Although the roles for PA in disease resistance were proposed (Kuć 1995), structures of the PA were found to be diverse, including flavonoid, isoflavonoid, diterpenoid, sesquiterpenoid, and indole (Grayer and Kokubun 2001), and precise studies to reveal the biological

M. Hasegawa, I. Mitsuhara, and S. Seo contributed equally to this work.

Corresponding author: Y. Ohashi; E-mail: yohashi@affrc.go.jp; Telephone: +81-29-838-7440; Fax: +81-29-838-7469. significance of each PA for resistance in individual experimental systems have been required.

In the monocot model plant rice, 15 PA have been isolated and characterized, including 14 diterpenes and one flavonoid PA, sakuranetin (Grayer and Kokubun 2001). The diterpene PA contain four structurally distinct types, phytocassanes A through $\mathrm{E}$, oryzalexins $\mathrm{A}$ through $\mathrm{F}$, momilactones $\mathrm{A}$ and $\mathrm{B}$, and oryzalexin $\mathrm{S}$, and their biosynthetic genes were recently identified (Kanno et al. 2006; Xu et al. 2007). Increases in PA in response to blast infection have been reported for momilactone A, oryzalexin E (Dillon et al. 1997), oryzalexin S (Kodama et al. 1992a), and phytocassanes A through E (Umemura et al. 2003) in resistant rice plants. A flavanone PA, sakuranetin, accumulated in resistant rice plants after blast fungus infection (Dillon et al. 1997) and UV irradiation (Kodama et al. 1992b). However, for better understanding on the role of rice PA in the resistance to blast fungus, levels of PA in blast fungus-inoculated resistant rice plants should be precisely compared with those in susceptible rice plants in the same genetic background. The detoxification and metabolism of PA in infected plants were thought to be important for the pathogenicity or virulence, or both, of fungi (Pedras and Ahiahonu 2005). However, evidence on the biosynthesis and fate of PA in infected rice plants was largely unknown. To study it, we used blast fungus (Magnaporthe oryzae) race 003 and rice plants (Oryza sativa L.) with and without the resistance gene $P i-i$ against the fungus. We first studied the accumulation and biosynthesis of major PA, momilactones A and B and phytocassanes A through $\mathrm{E}$, in fungus-infected resistant rice compared with those in susceptible rice. We also studied the effect of exogenous PA on blast growth and the detoxification of PA by the fungus, using momilactone A as a model PA. The present findings suggest that HR-associated rapid PA biosynthesis contributes to resistance in resistant rice plants, and delayed induction of PA biosynthesis in susceptible rice plants permits enhanced fungal growth, which confers detoxification of PA, inducing enhanced susceptibility to fungus.

\section{RESULTS}

The growth of blast fungus in resistant rice was severely restricted upon HR.

In the present experimental system, blast fungus race 003 can infect the susceptible rice strain Nipponbare $(\mathrm{N})$ without $\mathrm{HR}$, but blast infection induces resistance gene $P i-i$-mediated HR in resistant rice strain IL7, a near-isogenic line of Nipponbare into which the $P i-i$ gene has been introduced. Inoculated 

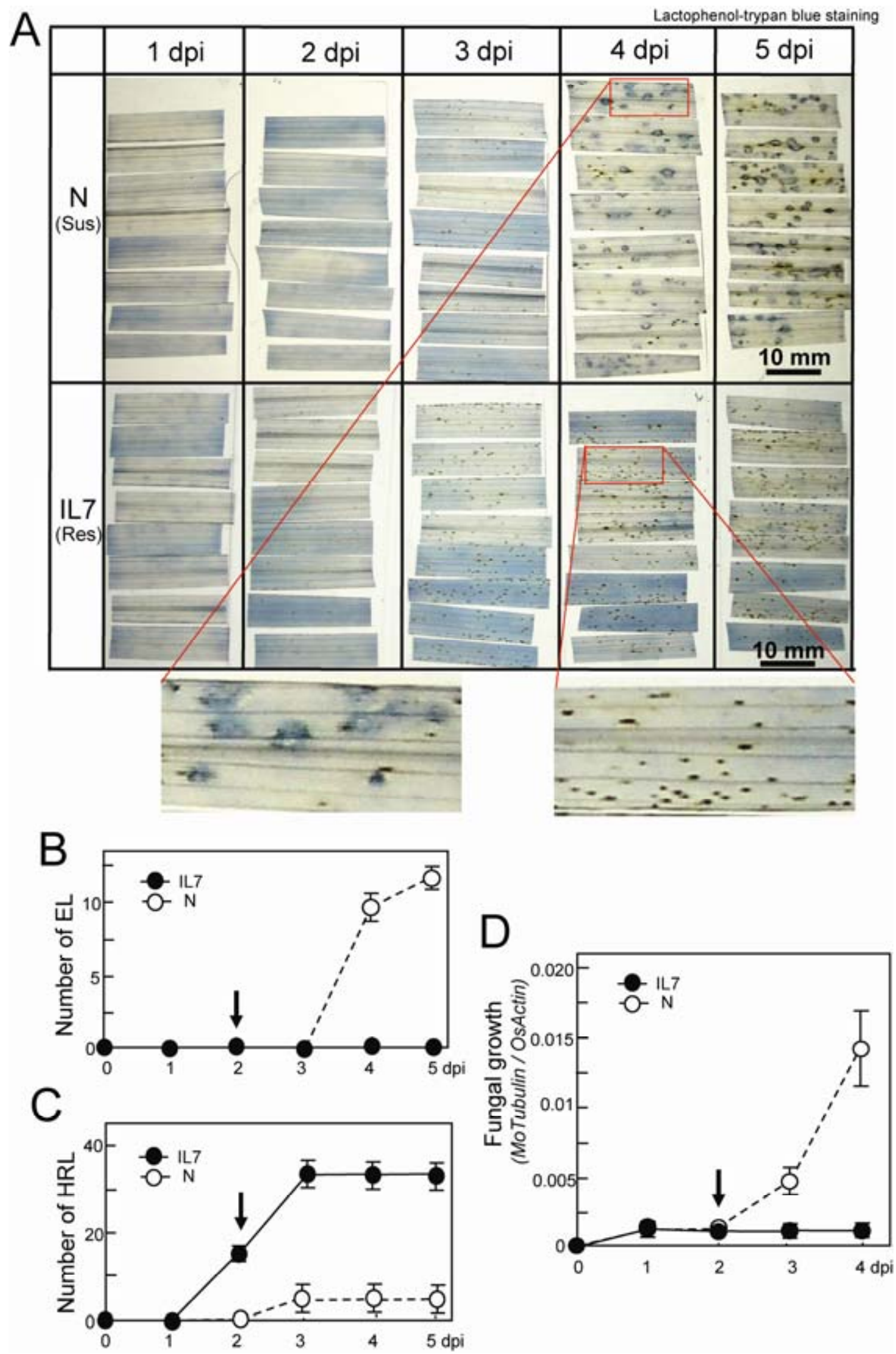

Fig. 1. Development of blast fungus-originated lesions in resistant and susceptible rice plants. A, Phenotypes of local lesions in the leaves of susceptible Nipponbare (N) and resistant IL7 rice plants. The fifth leaf of young seedlings harvested at the indicated day postinoculation (dpi) was cut into pieces $2 \mathrm{~cm}$ long and was subjected to lactophenol-trypan blue staining for the detection of fungal mycelia (Iwai et al., 2006). Bar $=10 \mathrm{~mm}$. B, The number of expanding lesions (EL) over $0.5 \mathrm{~mm}^{2}$ in a leaf piece $2 \mathrm{~cm}$ long. Closed circles = resistant IL7 plants; open circles = susceptible Nipponbare $(\mathrm{N})$ plants. Values are mean \pm standard error (SE) $(n=8)$. C, The number of hypersensitive response lesions (HRL) in a leaf piece $2 \mathrm{~cm}$ in length. In the case of Nipponbare (N), HRL indicate HRL-like lesions. Closed circles $=$ resistant IL7 plants; open circles $=$ susceptible Nipponbare $(\mathrm{N})$ plants. Values are mean \pm SE $(n=8)$. D, Content of blast fungus in leaves. Closed circles $=$ resistant IL7 plants; open circles $=$ susceptible Nipponbare $(\mathrm{N})$ plants. Values are mean $\pm \mathrm{SE}(n=3)$. 
rice leaves were subjected to lactophenol-trypan blue staining for clear observation of blast fungus-originated lesion phenotypes (Fig. 1A), because determination of the number and size of developed lesions in green leaves was difficult. In resistant IL7 leaves, small, necrotic HR lesions (HRL) with a dark brown color could be observed from 2 days postinoculation (dpi), and the number and the size of HRL seemed to be almost saturated at 3 dpi (Fig. 1C). In susceptible Nipponbare leaves, a smaller number of HR-like lesions were observed at 3 dpi (Fig. 1A and C), and some of these seemed to subsequently develop into large, expanding lesions (EL), which were observed at $4 \mathrm{dpi}$, increasing in number and size thereafter (Fig. $1 \mathrm{~A}$ and $\mathrm{B})$. EL were recognized by blue signal, which indicated spreading blast mycelia stained by trypan blue, while no blue signal was detected in and around HRL (Fig. 1A, lower magnified images). To quantify the amount of fungus in inoculated leaves, DNA was extracted and the content of fungal DNA was determined by real time polymerase chain reaction (PCR), using primers for the blast fungus tubulin gene normalized against a rice actin gene. Fungal DNA in IL7 was maintained at a lower level up to $4 \mathrm{dpi}$, after a small increase at 1 dpi, while that in Nipponbare increased considerably by 3 dpi and further at 4 dpi (Fig. 1D). The increase in fungal DNA content seemed to precede the increase in the number of EL that developed in Nipponbare leaves (Fig. 1B). These results indicate that the fungus rapidly multiplied in Nipponbare from $3 \mathrm{dpi}$, while the fungus in IL7 was already restricted by 1 to 2 dpi.

\section{Diterpene PA were accumulated after blast fungus inoculation more rapidly and highly in resistant plants than in susceptible plants.}

PA were extracted from inoculated leaves and were quantified by high-performance liquid chromatography coupled with electrospray ionization tandem mass spectrometry (HPLC-ESIMS/MS), using individual authentic chemicals as standards. A clear increase was observed in all PA in both resistant IL7 and susceptible Nipponbare after fungus inoculation (Fig. 2). At 2 to $4 \mathrm{dpi}$, the levels of all PA determined were higher in IL7 than in Nipponbare. At $2 \mathrm{dpi}$, at which HRL became visible, momilactone A was accumulated at a high level in IL7, while the PA in Nipponbare stayed almost at the basal level (Fig. 2A). Similarly, levels of momilactone B and phytocassanes A through $\mathrm{E}$ at 2 dpi seemed to be higher in IL7 than in Nipponbare (Fig. 2A and B). At 3 dpi, the amounts of momilactones A and B in IL7 were elevated to 2.4 and $1.2 \mu \mathrm{g}$ per gram of fresh leaf, which corresponds to about 500- and 1,000-fold of the basal levels, respectively, and those in Nipponbare were 0.5 and $0.1 \mu \mathrm{g}$ per gram of fresh leaf, respectively. Levels of phytocassanes A through $\mathrm{E}$ with an ent-cassane skeleton were also higher in IL7 and reached three- to fivefold of those in Nipponbare at $4 \mathrm{dpi}$ (Fig. 2B). The levels were highest in the order of phytocassanes A, B, C, E, and then, phytocassane D (data not shown). These data from three independent samples (Fig. 2) basically supported the previous preliminary results on the increase in rice PA after fungus inoculation (momilactone A, Dillon et al. 1997; phytocassanes A through E, Umemura et al. 2003) and more precisely and quantitatively showed the earlier and enhanced accumulation of seven PA at 2 to 4 dpi in resistant rice compared with susceptible rice.

\section{The genes involved in PA biosynthesis were more rapidly induced in resistant rice.}

To study whether the fungus-induced increases in PA shown in Figure 2 were accompanied by transcriptional activation, transcript levels of six genes related to PA biosynthesis were quantified. The four classes of rice diterpene cyclase genes were recently isolated and characterized (Kanno et al. 2006; Xu et al. 2007). Among them, OsCPS 2 and OsKSL7 are involved in phytocassanes synthesis, OsCPS4 and OsKSL4 in momilactones synthesis, and OSKSL10 and OsKLS8 in oryzalexin synthesis (Toyomasu 2008) (Fig. 3A). Using suspension-cultured rice cells, Shimizu and associates (2008) reported that the expression of OsCPS4, OsKSL4, OsCPS2, and OsKSL7 was induced within $12 \mathrm{~h}$ after chitin oligosaccharide elicitor treatment, followed by enhanced accumulation of phytocassanes and momilactones at 12 to 48 h. Quantitative real time PCR using gene-specific primer sets (Table 1) indicates that all genes analyzed here responded to fungus infection (Fig. 3B). The transcript level of OsCPS2 in IL7 at 2 to 4 dpi was increased similarly as in Nipponbare. However, the expression of $O s K S L 7$, which is involved in phytocassanes A through E synthesis, and that of OsCPS4 and OsKSL4, which confer momilactones synthesis, were increased transiently at $2 \mathrm{dpi}$ in IL7 but not in Nipponbare (arrows in Fig. 3B). OsKSL10 and OsKSL8 transcripts were also higher in IL7 than in Nipponbare at 2 dpi. But transcripts in Nipponbare were increased to similar or higher levels compared with those in IL7 at 3 dpi. At 4 dpi, transcripts for all six genes were elevated 10- to 50-fold compared with those at 0 to $1 \mathrm{dpi}$ in both IL7 and Nipponbare plants.

\section{Momilactone A treatment inhibited the growth of blast fungus in rice leaves.}

To study the effect of momilactone A in blast resistance in vivo, inoculated and detached fourth leaves of susceptible Nipponbare plants were placed in a glass tube at $1 \mathrm{dpi}$, to absorb the compound through the cut end (Fig. 4A). Developed lesion phenotypes observed at $4 \mathrm{dpi}$ after lactophenol-trypan blue staining indicate that the momilactone treatment reduced the size and number of EL (Fig. 4B). Moreover, fungal DNA in the leaves decreased to $30 \%$ of that in control leaves (Fig. 4B). These results indicate that exogenous momilactone A enhances blast resistance in rice leaves.

\section{The growth of blast fungus in vitro was inhibited by momilactone A treatment at 1 dpi but not thereafter.}

The effect of PA on the growth of blast fungus in vitro was studied using potato dextrose agar (PDA) medium containing 0.1 and $0.3 \mathrm{mM}$ momilactone A. When four small pieces (10 $\mathrm{mm}^{2}$ ) of mycelium were put on an agar plate $9 \mathrm{~cm}$ in diameter, they grew with time and reached a diameter of about 30 $\mathrm{mm}$ at 5 dpi (Fig. 5). In the presence of momilactone A, the growth of fungus was almost completely suppressed by $1 \mathrm{dpi}$ at both concentrations. The significance in the differences were confirmed by Student's $t$-test $(P<0.01)$. The growth restarted as early as $2 \mathrm{dpi}$ and thereafter continued at a similar high growth rate as the control. At $6 \mathrm{dpi}$, the size of mycelium circles became almost the same as the control (Fig. 5, right).

\section{Momilactone A was rapidly disappeared in the agar medium beneath fungal mycelium layer.}

The results shown in Figure 5 suggested rapid degradation of momilactone A by the fungus in the agar medium within 1 to 2 dpi. To study it, the concentration of momilactone $A$ in the part of the agar medium that was beneath the discs of the 15day-old fungal mycelium layer was determined (Fig. 6A). The concentration was $0.3 \mathrm{mM}$ at $0 \mathrm{~h}$, and rapid loss of the PA in the agar medium was found after being covered by the fungal layer (Fig. 6B). The levels were decreased by about half at 1 dpi and to $2 \%$ at $2 \mathrm{dpi}$, while those in the control media were maintained at almost the initial level until 2 dpi and decreased by only a small extent at 2 to $5 \mathrm{dpi}$. 


\section{Momilactone A was rapidly metabolized in fugal suspension culture.}

To study the fate of momilactone $\mathrm{A}$ in a suspension culture of blast fungus, the PA and the possible metabolites were analyzed by gas chromatography-mass spectrometry (GC-MS) after the addition of momilactone A. In the culture with a high content of fungus ( $3 \mathrm{mg}$ of protein equivalent per milliliter), the area of peak $\mathrm{A}$ at the retention time $21.1 \mathrm{~min}$ (for momilactone A) was decreased to about half after $0.1 \mathrm{~h}$ and to nearly null after 8 and $24 \mathrm{~h}$ of incubation (Fig. 7A). Instead of the decrease in momilactone $\mathrm{A}$, a possible metabolite at the retention time 18.3 min (peak B) was found after $0.1 \mathrm{~h}$ (Fig. 7A) but not after 8 and $24 \mathrm{~h}$ of incubation. Under a middle concentration of fungus ( $1 \mathrm{mg}$ of protein per milliliter), the area of peak $\mathrm{A}$ decreased to about one third and null at 4 and $8 \mathrm{~h}$, respectively, and that of peak B maximized at $4 \mathrm{~h}$ and decreased to null at 8 h (Fig. 7B, Total). In the culture with the lower concentration of fungus $(0.13 \mathrm{mg} / \mathrm{ml})$, peak $B$ was still found at $8 \mathrm{~h}$ but not $24 \mathrm{~h}$ (data not shown). These results indicate that momilactone A was converted to the peak B substance and the conversion activity depended on the concentration of the fungus. Another candidate of momilactone A metabolite, a peak at retention time $18.8 \mathrm{~min}$, was detected (Fig. 7A, open arrowhead), although the level was only $5 \%$ of that of peak B. In the absence of blast fungus, the level of momilactone A was maintained for $24 \mathrm{~h}$ without any detectable decrease and no peak was detected at $18.3 \mathrm{~min}$. The mass spectrum of peak B showed a probable molecular ion at $\mathrm{m} / \mathrm{z}$ 286. Since the molecular weight of momilactone $\mathrm{A}$ is 314 , the novel peak B substance at 18.3 min would be a possible metabolite of momilactone A. The structure of the peak B molecule is now under investigation.

To study further, the PA and the possible fungus metabolites were analyzed separately from those in the culture medium. The fungus precipitate and the supernatant medium in a suspension culture (1 $\mathrm{mg}$ of fungal protein per milliliter) were treated with $70 \%$ methanol at 4 and $8 \mathrm{~h}$ after addition of 0.3 $\mathrm{mM}$ momilactone $\mathrm{A}$ in the final concentrations, and the extracts were submitted to GC-MS analysis. Unexpectedly, momilactone $\mathrm{A}$ was found in the fungus fraction at 4 and $8 \mathrm{~h}$ but not in the medium fraction. In contrast, only a low level of peak B substance was found in the fungus and a considerable level was found in the medium (Fig. 7B). In the culture medium, momilactone A was stable for $24 \mathrm{~h}$. Incubation with the boiled fungal suspension culture resulted in no detectable loss of momilactone A, indicating the heat treatment abolished the enzymatic activity leading to metabolization of the PA. These results suggest that momilactone $\mathrm{A}$ was first incorporated and converted to the peak B substance in the fungus and the metabolite was transferred to the medium.

Next, on the same samples used for the results shown in Figure $7 \mathrm{~B}$, antifungal activity was determined via the inhibitory effect of germ tube growth from blast spores, using an inverted microscope. In extracts from the fungal precipitates, growth of germ tubes was considerably inhibited (Fig. 7C and D). The inhibition at $8 \mathrm{~h}$ was clearly less than that at $4 \mathrm{~h}$, reflecting the data of momilactone A in precipitates (Fig. 7B). In the medium fraction of fungal suspension at $4 \mathrm{~h}$, in which peak B substance but not momilactone A was contained, considerable antifungal activity was found, however, in a medium fraction at $8 \mathrm{~h}$, in which no peak B and momilactone A was found, no detectable antifungal activity was found (Fig. 7C and D). The results illustrated in Figure 7 indicate that the inhibitory activity at $4 \mathrm{~h}$ in the medium depended on one or both the peak B substance or unknown metabolites of the PA and lead to the supposition that the activity of the peak B substance was similar to or a little less than that of momilactone A. Further studies using isolated peak B molecules are necessary to assess it.

\section{DISCUSSION}

More prompt and higher levels of accumulation of seven diterpene PA, i.e., momilactones A and B and phytocassanes A through E, was found in resistant rice plants than in susceptible rice plants after blast fungus inoculation (Fig. 2). In resistant IL7 plants, increase in PA at the early infection time 2 dpi
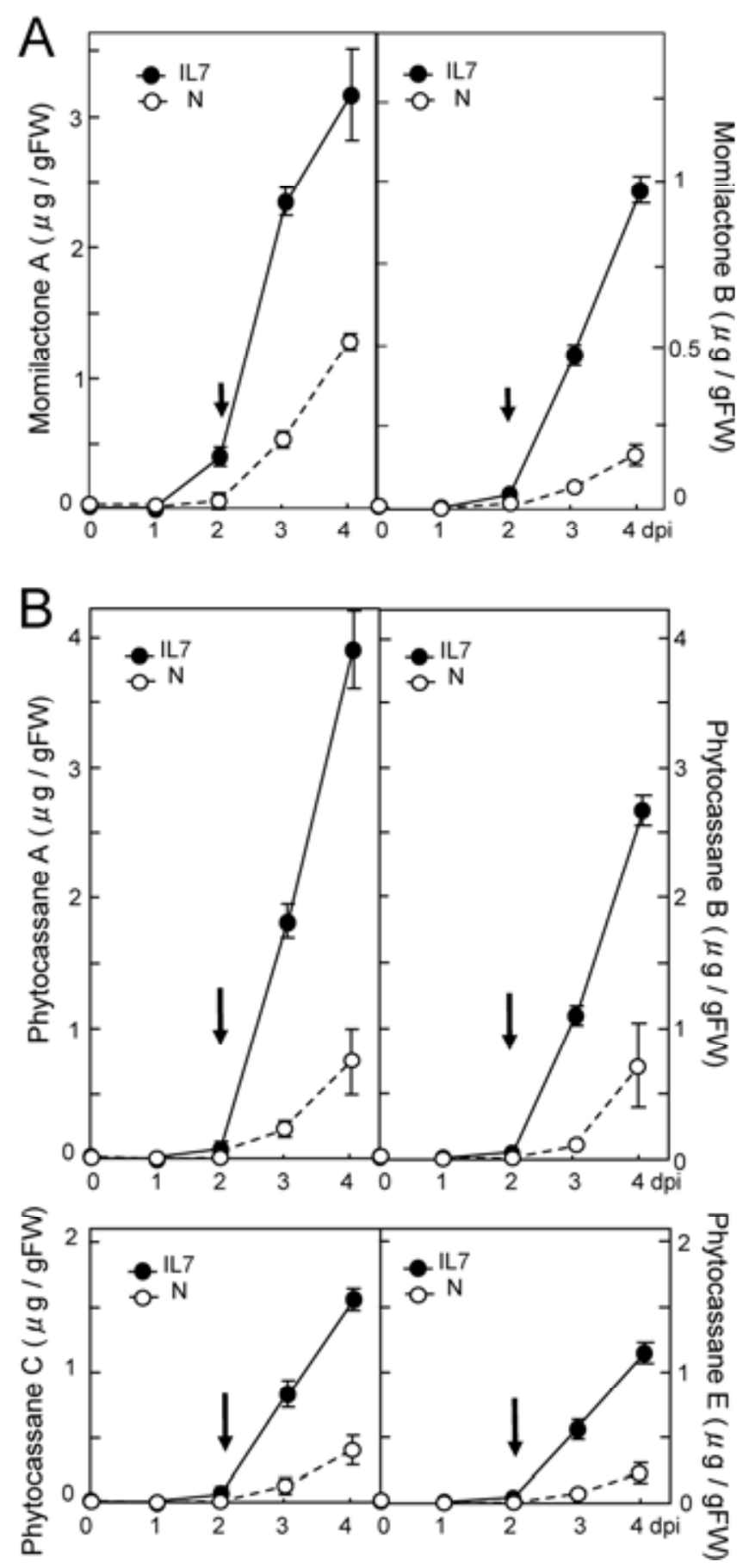

Fig. 2. Accumulation of diterpenoid phytoalexins (PA) in blast fungusinoculated susceptible and resistant rice leaves. Endogenous contents of PA are shown as micrograms per gram of fresh leaf with mean \pm standard error of three independent samples. Closed circles $=$ resistant rice, IL7; open circles $=$ susceptible rice, Nipponbare $(\mathrm{N})$. Arrows indicate 2 days postinoculation, when necrotic hypersensitive response lesions started to be visible by the naked eye in IL7. A, Momilactones A and B, B, phytocassanes A, B, C, and E. Phytocassane D was induced with a similar induction mode, but all values were lower than the other phytocassanes shown (data not shown). 
A

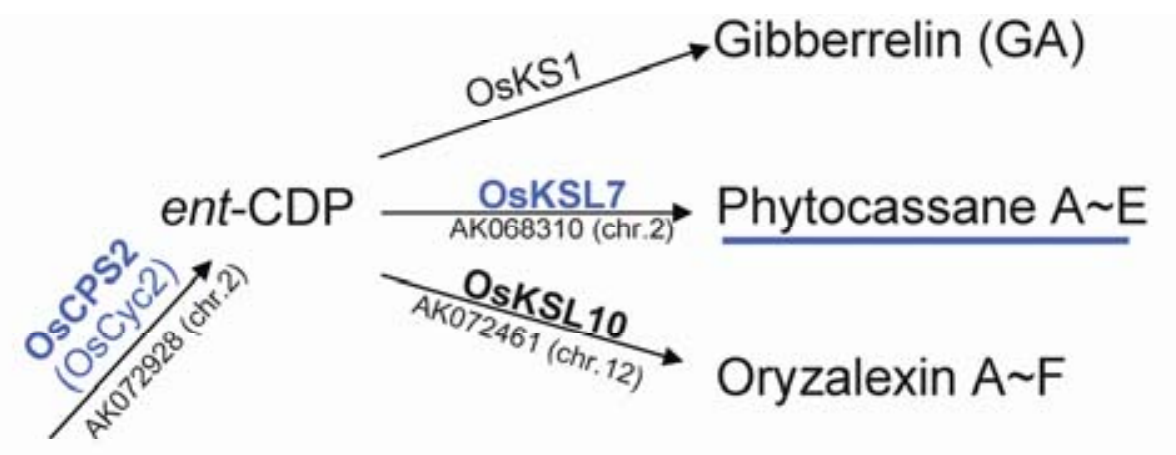

\section{GGDP}
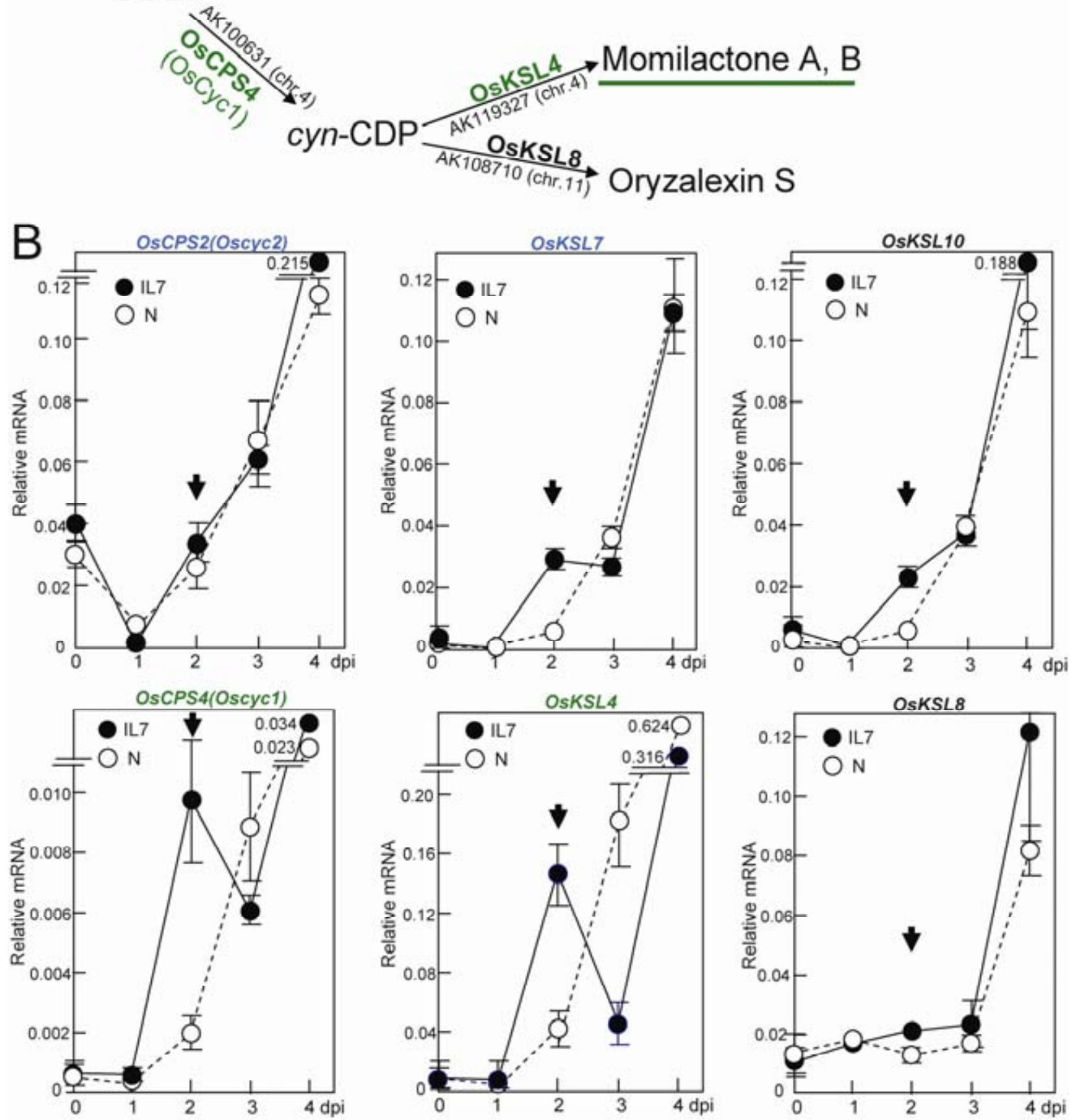

Fig. 3. Induced expression of genes for diterpene phytoalexin (PA) synthesis in blast fungus-inoculated susceptible and resistant rice leaves. A, Proposed biosynthetic pathways for rice diterpene PA (Toyomasu 2008). B, Transcript levels of genes for rice diterpene PA synthesis in fungus-inoculated susceptible rice Nipponbare (N) (open circles) and resistant rice IL7 (closed circles) were determined by quantitative polymerase chain reaction. Data from three independent samples are shown mean \pm standard error. Arrows indicate 2 days postinoculation, when necrotic hypersensitive response lesions were initially observed in IL7. 
was accompanied by transient induction of PA biosynthetic genes (Fig. 3B). Because the rapid response in IL7 was not found in susceptible Nipponbare, it would be dependent on the $P i-i$ resistance gene. Interestingly, at the later infection stage 4 dpi, transcripts of PA biosynthetic genes in susceptible plants were accumulated to levels as high as or higher than in resistant plants. This may be due to increased pathogen ingress and, thus, to more tissue being exposed to the pathogen, giving stronger reaction in the end. This supports the effectiveness of rapid responses in the beginning of the interaction. In spite of the high transcript levels, accumulation of PA in susceptible plants was lower (Fig. 3B), suggesting possible enhanced degradation of PA by the fungus. In fact, momilactone A was rapidly metabolized and detoxified by blast fungus in vitro (Figs. 6 and 7). We found that a rice flavonoid PA, sakuranetin, was similarly metabolized by the fungus in vitro (unpublished data). The detoxification of PA from the family Graminaceae has not been shown previously. However, several reports indicated that PA are detoxified by fungal pathogens that infect dicot plants. For example, camalexin was metabolized by Rhizoctonia solani (Pedras and Khan 1997) and Sclerotinia sclerotiorum (Pedras and Ahiahonu 2002) to less toxic products, and a pea PA, pisatin, was metabolized by fungal pathogen Nectria haematococca to (+)-6a-hydroymaackiain via demethylation (VanEtten et al. 1975). The data in Figures 6 and 7 suggest that blast fungus has a massive activity to metabolize $\mathrm{PA}$; the antifungal activity in the suspension culture with 0.3 $\mathrm{mM}$ momilactone A was decreased with time, depending on the presence of the fungus, and the decrease reflected the disappearance of momilactone $\mathrm{A}$ and the possible metabolites, in which antifungal activity was found.

Notably, momilactone A was incorporated into the fungus at first, and it then disappeared from the fungus over time. Instead, a possible metabolite with retention time $18.3 \mathrm{~min}$ appeared as peak B in the medium. Although the level of the antifungal activity of the Peak B substance is not precisely known at this time, it would be true that momilactone A was metabolized and detoxified by blast fungus. PA other than momilactone A could also be detoxified by the fungus, because their accumulation patterns (Fig. 2) and the expression patterns of the biosynthetic genes (Fig. 3) were similar to those of momilactone A. Detoxification of PA could be a counter-defense strategy of invading parasites. The pathogen that can effectively detoxify PA could grow in the host plant, resulting in susceptibility. The levels of PA, which are mainly determined by the balance of biosynthesis by the host plant and detoxification by the fungus, may strongly contribute to disease resistance. The $\mathrm{ABC}$ transporter BcatrB from Botrytis cinerea was proposed to be a virulence factor that increases tolerance of the pathogen by control of the active transport of an Arabidopsis PA camalexin (Stefanato et al. 2009). Although the actual substrate transported was not clearly shown, the three ABC transporters of $M$. oryzae that are reportedly required for virulence (Gupta and Chattoo 2008; Sun et al. 2006; Urban et al. 1999) are possible factors that increase the susceptibility of rice plants to the fungus. For survival in infected plants, blast fungus may have evolutionally acquired the ability for PA detoxification, and resistant host plants might produce PA faster in gene-for-gene interactions to overcome the tactic of fungus (Fig. 8). Rapid PA production would be one of the many tools in the gene-for-gene toolbox and confers to resistance in concert with other defense mechanisms in resistant plants. Further, all the rice PA may have the cooperative effect on fungitoxicity, and may even be synergistic.

The concentrations of momilactone $\mathrm{A}$ and $\mathrm{B}$ giving $50 \%$ inhibition of germ tube growth were 5 and $1 \mu \mathrm{g} / \mathrm{g}$, respec- tively (Cartwright et al. 1977). Although the levels of momilactones A and B detected in whole leaves of IL7 at 3 dpi (mean 2.4 and $1.2 \mu \mathrm{g}$ per gram of fresh leaf, respectively) seem to be insufficient for an effective restriction of fungus, the local concentrations around HRL may reach much higher levels. Previous reports suggested that the local amount of
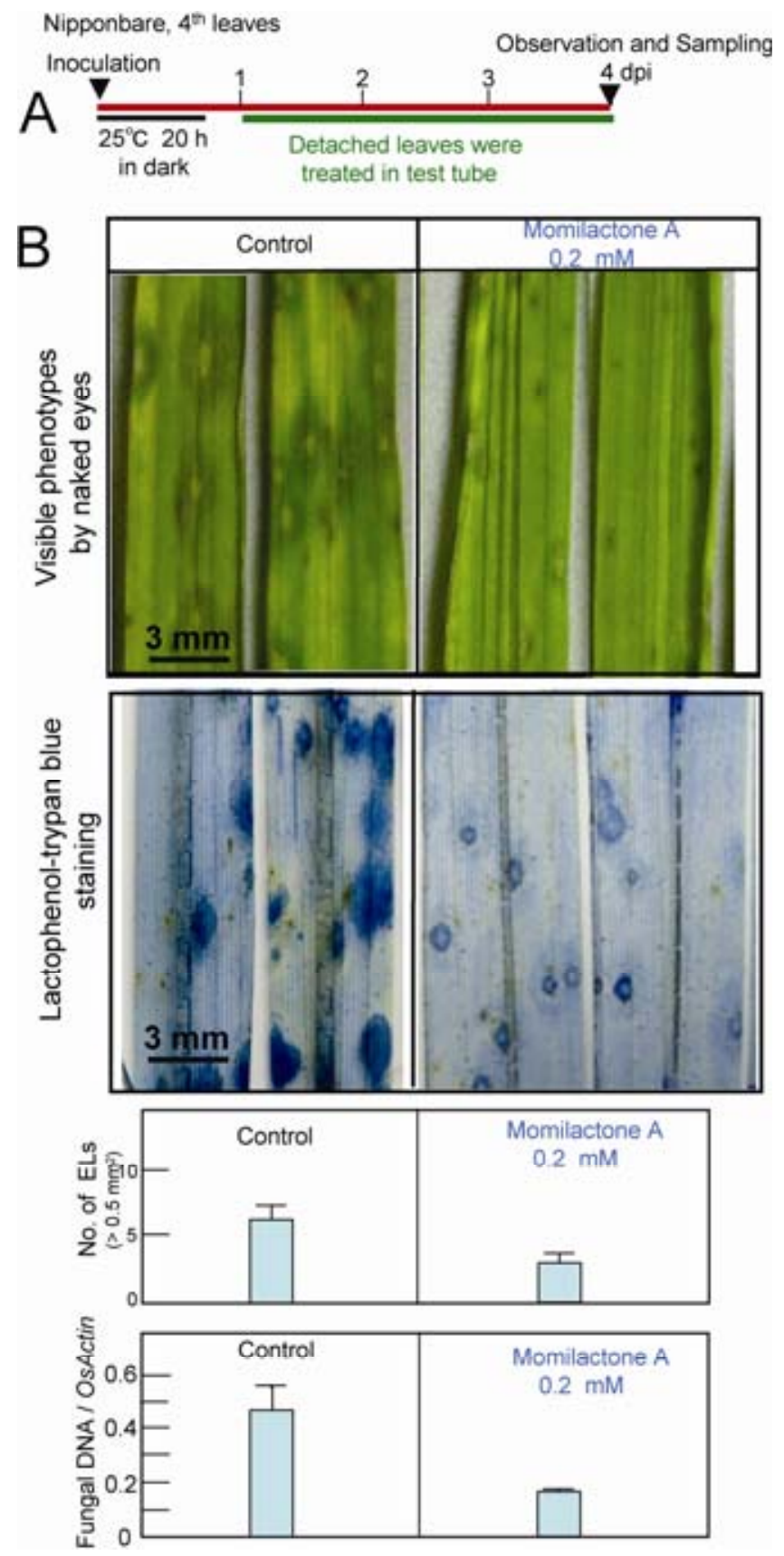

Fig. 4. Exogenous momilactone A suppressed fungal growth in detached leaves. A, Timeline of the assay with detached leaves. B, Inoculated fourth leaves of Nipponbare were detached at 1 day postinoculation (dpi), and were stood in a glass tube containing $10 \mathrm{ml}$ of $0.5 \%$ methanol (Control) or $0.5 \%$ methanol with momilactone A at $0.2 \mathrm{mM}$ (Momilactone A). Upper panel, leaves with expanding lesions (EL) at $4 \mathrm{dpi}$. Second panel, phenotypes of EL after lactophenol-trypan blue staining. Third panel, number of EL in a leaf piece $20 \mathrm{~mm}$ in length. The mean number of EL larger than $0.5 \mathrm{~mm}^{2}$ were counted after lactophenol-trypan blue staining and are shown with $\mathrm{SE}(n=10$ to 12$)$. Bottom panel, fungal DNA was semiquantified by quantitative polymerase chain reaction using Mo-tubulin and was normalized with the transcript levels of a rice actin gene. Repeated experiments using inoculated fifth leaves indicated similar results. 
PA around pathogen-induced lesions was higher than the mean value in leaves. i) High levels of Arabidopsis PA camalexin were found at the infection site or in proximity to pathogen-induced lesions with strong localized induction of camalexin biosynthetic genes, while leaf areas that did not show disease symptoms were PA deficient (Schuhegger et al. 2007). ii) Oat PA avenalumin accumulated only in infected regions but not in the adjacent uninoculated areas (Mayama et al. 1982). iii) The synthesis of sorghum PA 3-deoxyanthocyanidin flavonoids occurred as a site-specific response to fungal ingress (Snyder and Nicholson 1990). iv) A potato PA, rishitin, accumulated exclusively in infected dead cells as a result of secretion from the adjacent live cells in which rishitin was actively synthesized (Tomiyama 1980). v) Phytocassanes A through E accumulation was most abundant at the edges of necrotic lesions in blast infected rice leaves (Umemura et al. 2003). According to the postulation that much higher levels of PA accumulate locally at or around pathogen-induced lesions, we calculated the predicted local PA concentration around HRL in IL7. As the leaf area occupied by HRL at 3 dpi (Fig, 1A) was roughly calculated to be $0.02 \%$ of the total leaf area, PA levels at or around HRL were estimated at about 5,000 -fold higher than the mean values in the leaves shown in Figure 2, corresponding to 12 and $6 \mathrm{mg}$ per gram of fresh leaf for momilactone $\mathrm{A}$ and $\mathrm{B}$, respectively. The concentrations of phytocassanes A, B, C, D, and E in IL7 at 3 dpi were increased to mean $1.8,1.1,0.9,0.3$, and $0.5 \mu \mathrm{g}$ per gram of fresh leaf, respectively, and concentrations giving 50\% inhibition of germ tube growth were 5, 1.5, 3, 10 (Koga et al. 1995), and $6 \mu \mathrm{g}$ (Koga et al. 1997) per gram of fresh leaf, respectively. The calculated concentrations of phytocassanes A through E around HRL at $3 \mathrm{dpi}$ are 9 to $1.5 \mathrm{mg}$ per gram of fresh leaf. If accurate, the predicted local concentrations of PA around HRL may be enough to restrict the fungal growth in IL7. Further, multiple PA at increased levels may have a cooperative effect on the fungitoxicity.

Based on these results, predicted responses of resistant rice to blast fungus infection were compared with those of sus-

Table 1. Primers for polymerase chain reaction

\begin{tabular}{lll}
\hline Gene name & \multicolumn{1}{c}{ Left primer } & \multicolumn{1}{c}{ Right primer } \\
\hline OsCPS2 & \multicolumn{1}{c}{ AACAAGAGTTGTGTCTACCAGCATG } & ACAAATATCGACTAAATTCATCTCACTTTG \\
Os $K S L 7$ & TGTTCTACTACCAGACCGACGGATTC & CTGAGTTGAAGTGGCTCGTTGATGAC \\
Os $K S L 10$ & CGTGAGTGCATGTGAGAGTCCATC & TTCCATCAAACTTTCTCCTCAATTTCCAAG \\
OsCPS4 & AAAACACGAGGTACACCACAACAAAC \\
Os $K S L 4$ & CCCCACCTCCACTACCAATTCCC & GGAAACCTGAATAGTTTAAGCACCGTTC \\
Os $K S L 8$ & GCTTGTTTGCGAGGACATTCCATC & ATATGCGCGGGTTTCTTCCTAGTTTTAG \\
\hline
\end{tabular}

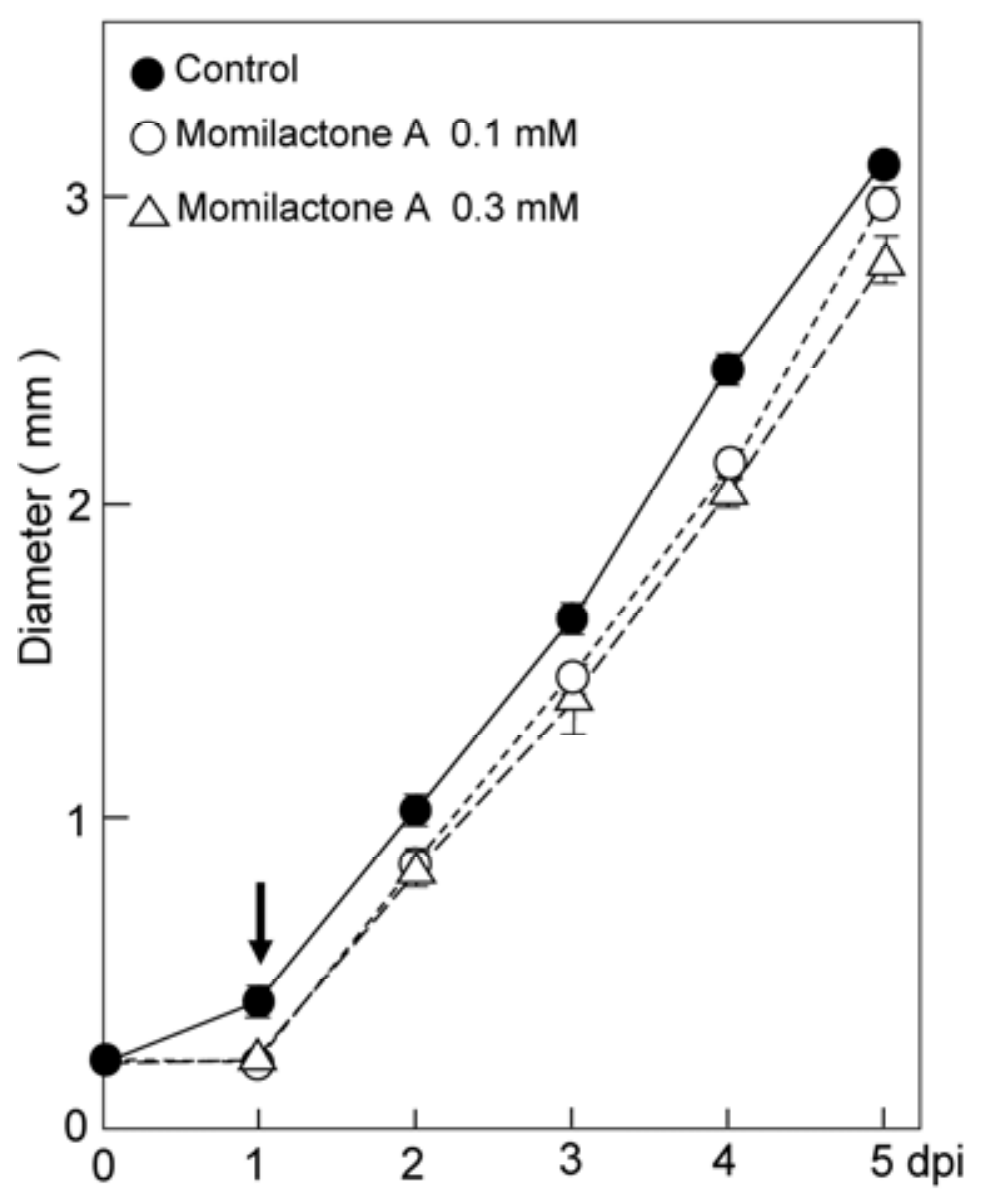

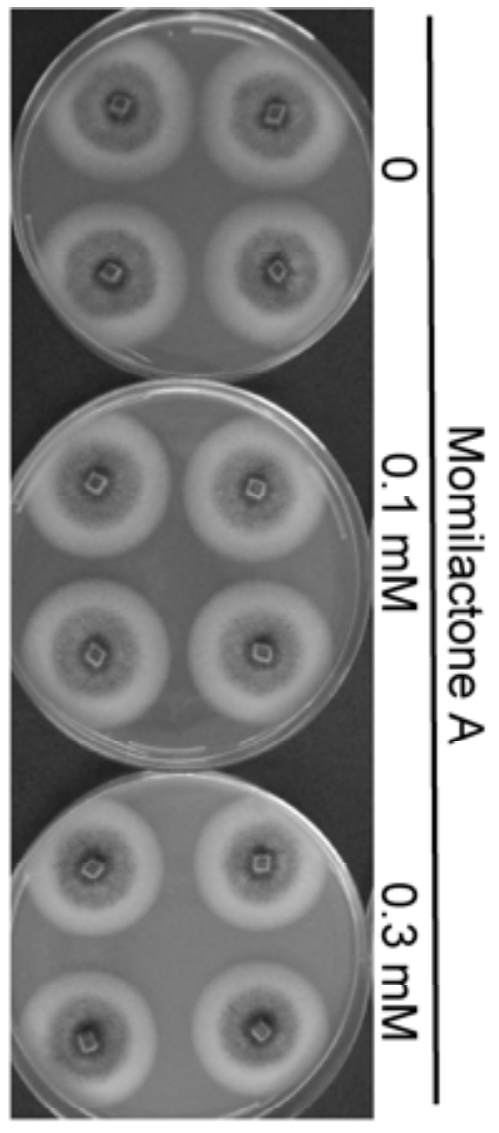

$6 \mathrm{dpi}$

Fig. 5. Effect of momilactone A on the growth of blast fungus in agar medium. Four pieces of 15-day-old mycelial layers of Magnaporthe oryzae were incubated on potato dextrose agar medium with $0,0.1$, and $0.3 \mathrm{mM}$ momilactone $\mathrm{A}$, and the diameters of the disc layers were measured at the indicated time points. Values given are mean \pm standard deviation of four independent measurements. The right panel shows a photograph at 6 days postinoculation. 
ceptible rice plants (Fig. 8). Using the same experimental system, Kuroda and associates (2006) have reported that no significant difference was found in conidial behavior on inoculated leaves immediately following the spraying of conidia suspension. They also showed that a defense-related rice $M A P K$ gene, which is an ortholog of transcriptionally activated tobacco WIPK (Seo et al. 1995), and peroxidase gene $R 2184$, whose expression was induced rapidly by blast fungus infection and wounding (Sasaki et al. 2004), were similarly induced at $1 \mathrm{~h}$ after inoculation in both Nipponbare and IL7, as predicted by the basal resistance response. Iwai and associates (2006) used the same system and reported that ethylene-cyanide biosynthesis, which may contribute to the HR-mediated restriction of blast fungus, was increased transiently at 2 to 3 dpi for the resistant rice-specific response. In this paper, we showed that momilactones $\mathrm{A}$ and $\mathrm{B}$ and five phytocassanes start to accumulate along with increased transcript levels of the biosynthetic genes in resistant rice at 2 to $3 \mathrm{dpi}$ but not in susceptible rice. Locally concentrated PA levels around HRL (Umemura et al. 2003) may effectively restrict fungal growth in resistant rice, but the restriction may fail in susceptible rice, probably because of substantially lower PA levels. In later host responses ( 3 to $4 \mathrm{dpi}$ ), the fungus multiplies in susceptible rice, and the induced expression of PA biosynthetic genes at 3 to 4 dpi may not be in time to accumulate much PA because PA are detoxified by the increased fungal biomass. In resistant rice, PA continue to increase with no significant detoxification because fungal growth is restricted by 2 to 3 dpi. Thus, rapid restriction of the fungus around initial infection sites may be critically important for early resistance responses in IL7.

The findings obtained here emphasized the importance of PA in HR-mediated disease resistance and may help in understanding the dynamic host-parasite battle for survival on PA via their biosynthesis and detoxification. For the next research study of the role of PA, we are interested in the mechanism of HR-dependent induction of PA biosynthetic genes as well as the analysis of PA-deficient plants.

\section{MATERIALS AND METHODS}

\section{Plant materials.}

For susceptible and resistant rice (Oryza sativa) cultivars, Nipponbare and IL7 (Ise and Horisue 1988), respectively, were used. IL7 is a near-isogenic line of Nipponbare into which $R$ gene $P i-i$ against blast fungus (M. oryzae) race 003 (isolate Kyu89-241; Yamada et al. 1976) has been introduced. Plants were grown in soil (Bonsol No.1; Sumitomo Chem. Ltd. Osaka, Japan) in a growth chamber under light $\left(5 \mu \mathrm{mol} \mathrm{m} \mathrm{m}^{-2} \mathrm{~s}^{-1}\right)$ for $14.5 \mathrm{~h}$ at $28^{\circ} \mathrm{C}$ and for $9.5 \mathrm{~h}$ in the dark at $25^{\circ} \mathrm{C}$, and one or both the fourth and fifth leaves of 14- to 16-day-old seedlings were used as plant materials.

\section{Inoculation with blast fungus and \\ measurement of local lesions.}

Blast fungus race 003 was grown on oatmeal medium (Difco, Detroit) for 2 weeks, and conidia were induced under BLB light (FL20S BLB; Toshiba, Tokyo) for 2 days at $25^{\circ} \mathrm{C}$. A conidia suspension $\left(1 \times 10^{5}\right.$ conidia $\left.\mathrm{ml}^{-1}\right)$ containing $0.05 \%$ Tween 20 was sprayed on the rice seedlings, and incubation was carried out under high humidity in the dark for $20 \mathrm{~h}$ at $25^{\circ} \mathrm{C}$ for effective infection, and then, the seedlings were moved to a chamber at $25^{\circ} \mathrm{C}$ under a 16 -h light and 8 -h dark cycle. The number and size of lesions that developed were analyzed after taking closeup photographs of detached leaves alongside a standard ruler. Leaves were cut into 20-mm lengths for lactophenol-trypan blue staining, which was performed after treatment with ethanol (Iwai et al. 2006). The number and size of enlarged susceptibletype EL were analyzed by detecting the blue signal of fungal mycelium stained by trypan blue.

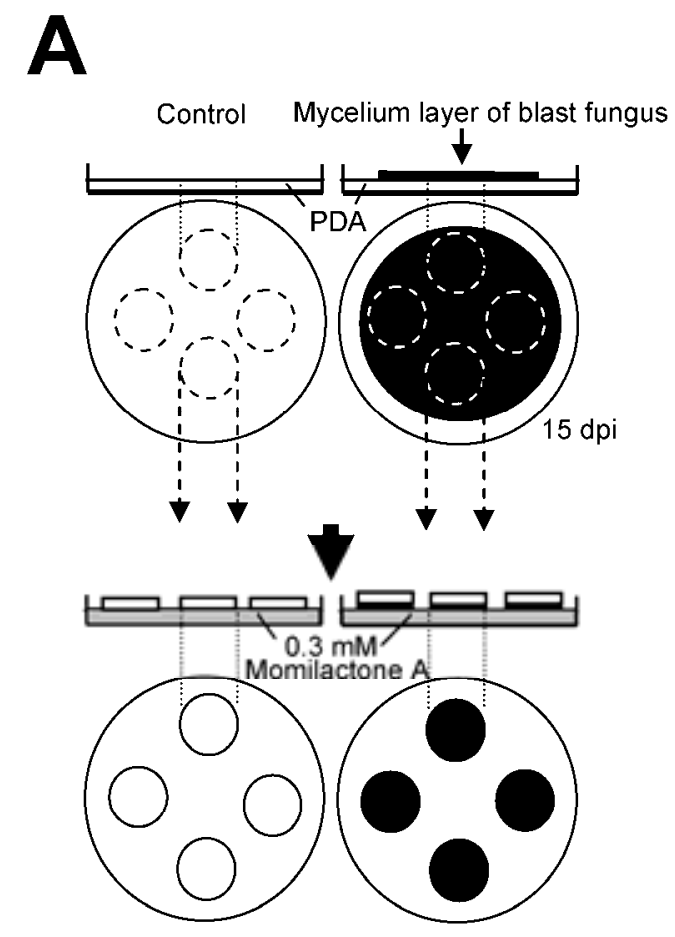

Fig. 6. Disappearance of momilactone A in the agar medium in direct contact with the mycelium layer. A, Illustration of the experimental method. B, Discs $20 \mathrm{~mm}$ in diameter of 15-day-old mycelial layers of Magnaporthe oryzae on potato dextrose agar medium were put upside down on the agar medium containing a 3-mm-thicklayer of $0.3 \mathrm{mM}$ momilactone A. The agar medium beneath the mycelial disc layer was collected at the indicated time points, and the content of momilactone A was determined. The momilactone A values shown are ratios to the start point mean \pm standard deviation of four independent measurements. Closed circles = with mycelial layer; Open circles = with control agar layer without fungus. 
A

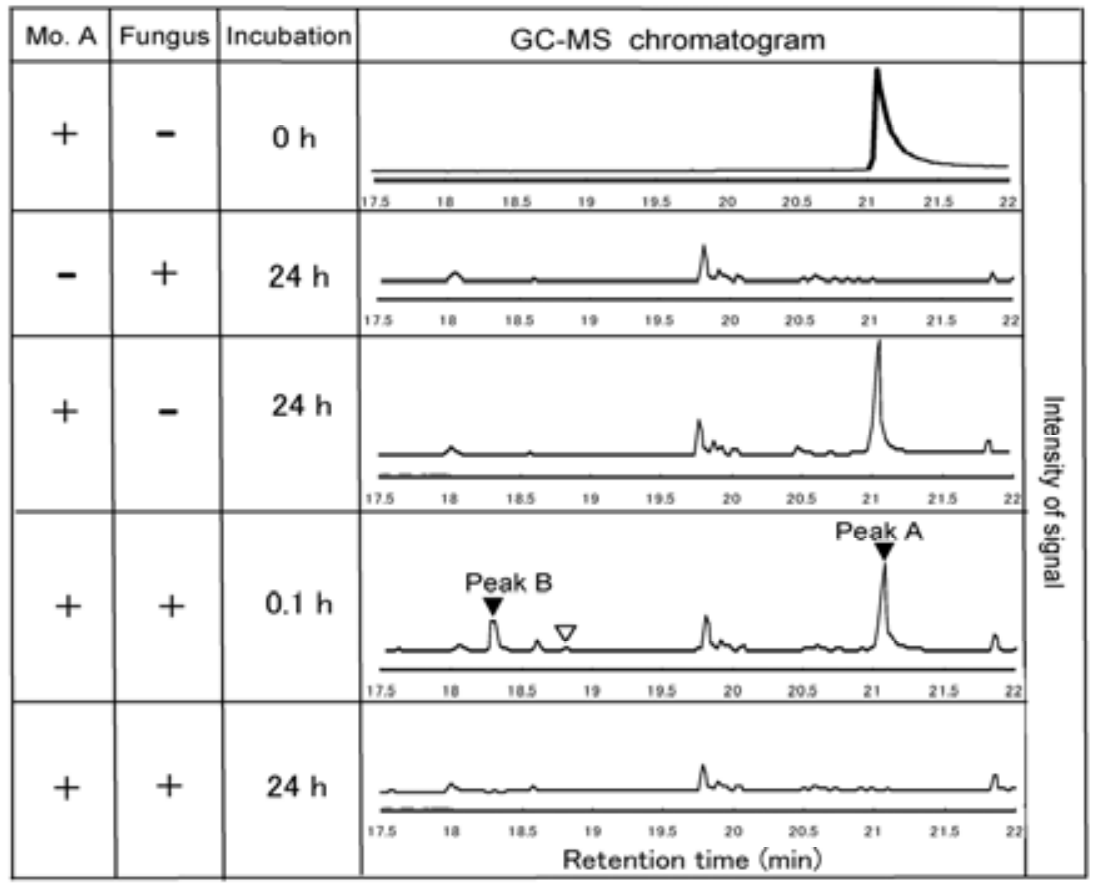

D

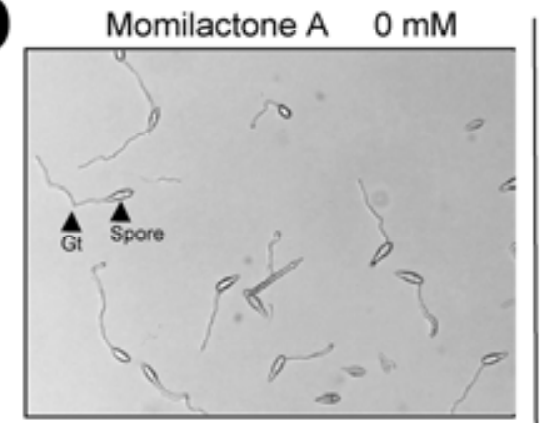

Momilactone A $\quad 0.3 \mathrm{mM}$

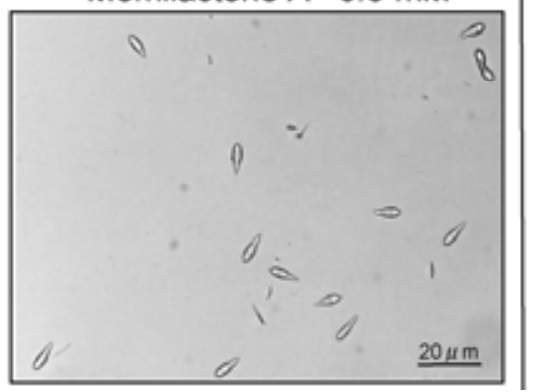

B

- Peak A (Mo. A)

- Peak B (18.3 min)
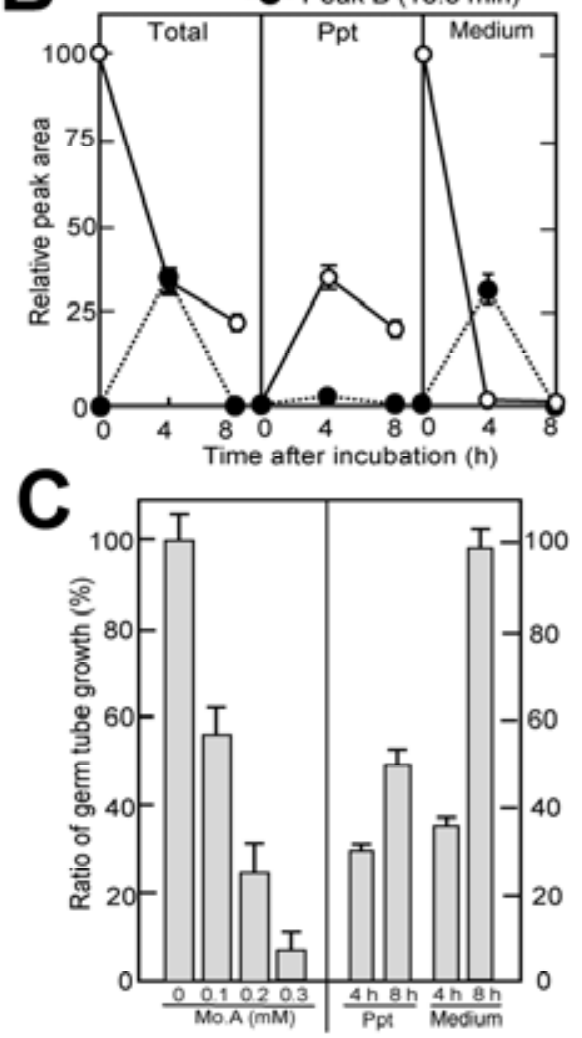

$4 \mathrm{~h}$
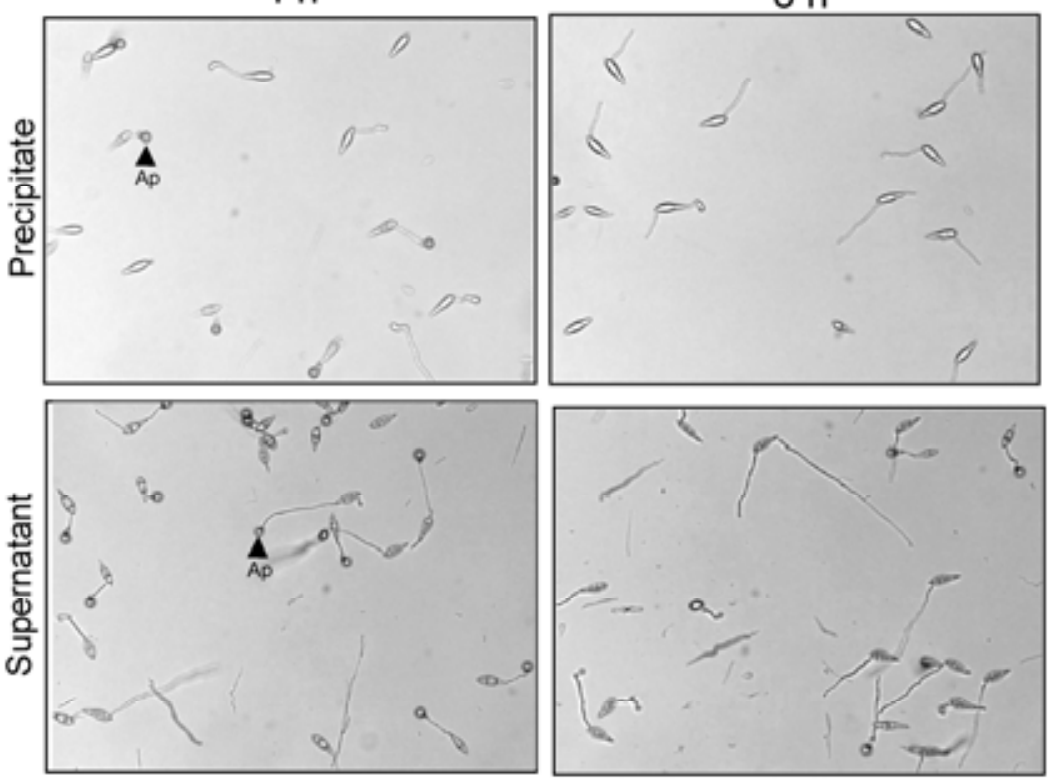

Fig. 7. Detoxification of momilactone A in suspension culture of blast fungus. Momilactone A (Mo. A) was added to 1 ml of potato dextrose (PD) liquid culture with or without blast fungus, and the phytoalexins and the possible metabolites were extracted with $70 \%$ methanol after incubation for $0,0.1,4,8$, and $24 \mathrm{~h}$. A, Gas chromatographic-mass spectrometry chromatograms. Total ion chromatogram at a scan range of $m / z$. 55 to 700 . Peak A corresponds to momilactone A and peak B to the possible metabolite with retention time $18.3 \mathrm{~min}$. The open triangle indicates the signal for retention time 18.8 min. B, Relative peak area of peak A (open circle) and peak B (closed circle) substances. The area of 0.3 mM momilactone A was presumed as 100 . Values were mean \pm standard deviation of three to six independent measurements. $\mathbf{C}$, Effect on germ tube growth of the extracts with $70 \%$ methanol from the precipitates and supernatants of fungal cultures. Values were mean \pm standard error of three to six independent measurements. Left, standard data on momilactone A. Right, relative values in the extracts. The samples were the same as those used in B. Values are mean \pm standard error of three independent data, each of which was calculated using five sets of independent photographs containing 20 to 50 spores by an inverted microscope (DIAPHOTO TMD, Nikon, Tokyo). Some of the photographs are shown in D. D, Photographs of germ tube elongation from blast spores. Freshly prepared blast spores $\left(4 \times 10^{3}\right)$ were suspended in $100 \mu \mathrm{l}$ of PD medium containing each extract to be tested, and the mixture was put in the center hole (10 mm in diameter) of a glass-bottom dish ( 35 mm in diameter). Photographs were taken after incubation at $20^{\circ} \mathrm{C}$ for $20 \mathrm{~h}$ in the dark. In both precipitate and supernatant samples at $4 \mathrm{~h}$, rate of appressorium formation was higher than that of 0 or $8 \mathrm{~h}$, although the reason is not clear. $\mathrm{Sp}=$ spore, Ap $=$ appressorium, $\mathrm{Gm}=\mathrm{germ}$ tube. $\mathrm{Bar}=20 \mu \mathrm{m}$. 


\section{Quantification of PA.}

For PA quantification in blast fungus-inoculated leaves, inoculated leaves were detached after indicated the time periods and were cut into 5 -mm lengths, and $0.15 \mathrm{~g}$ of each was frozen at $-80^{\circ} \mathrm{C}$ until use. A leaf sample was mixed with 40 volumes of $70 \%$ methanol and was heated for $5 \mathrm{~min}$ in a long glass tube with a screw cap. The extract was transferred to a new tube, and the residue was re-extracted twice with 20 volumes of $70 \%$ methanol. The combined extracts were concentrated to dryness. The residue was resuspended in $0.5 \mathrm{ml}$ of methanol and was subjected to HPLC-ESI-MS/MS analysis under the same conditions as described previously (Shimizu et al. 2008). For PA quantification in PDA, medium containing momilactone A (initial concentration $0.3 \mathrm{mM}$ ) was mixed with 9 volumes of methanol. The PA was extracted by shaking for $120 \mathrm{~min}$, and after centrifugation for $10 \mathrm{~min}$ at $10,000 \times \mathrm{g}, 10 \mu \mathrm{l}$ of the supernatant was subjected to HPLC-ESI-MS/MS as described above.

\section{Quantification of M. oryzae DNA.}

Fungal DNA was extracted from inoculated leaves using ISOPLANT (Nippon Gene, Toyama, Japan) and was quantified by real time PCR according to the method of Qi and Yang (2002), using two specific primer pairs that were designed based on the $3^{\prime}$ noncoding region of a tubulin gene in $M$. oryzae (forward, 5'-GGGATGATGGTGGTGGAGGAC-3' and reverse, 5'-GCCAGGTGCTTAGGACGAAAC-3'). These data were normalized with the DNA amount of a rice actin gene (AK060893), which was quantified using the following primers (forward, 5'-GAGTATGATGAGTCGGGTCCAG-3' and reverse, 5'-ACACCAACAATCCCAAACAGAG-3'). The real-time PCR was conducted as described below.

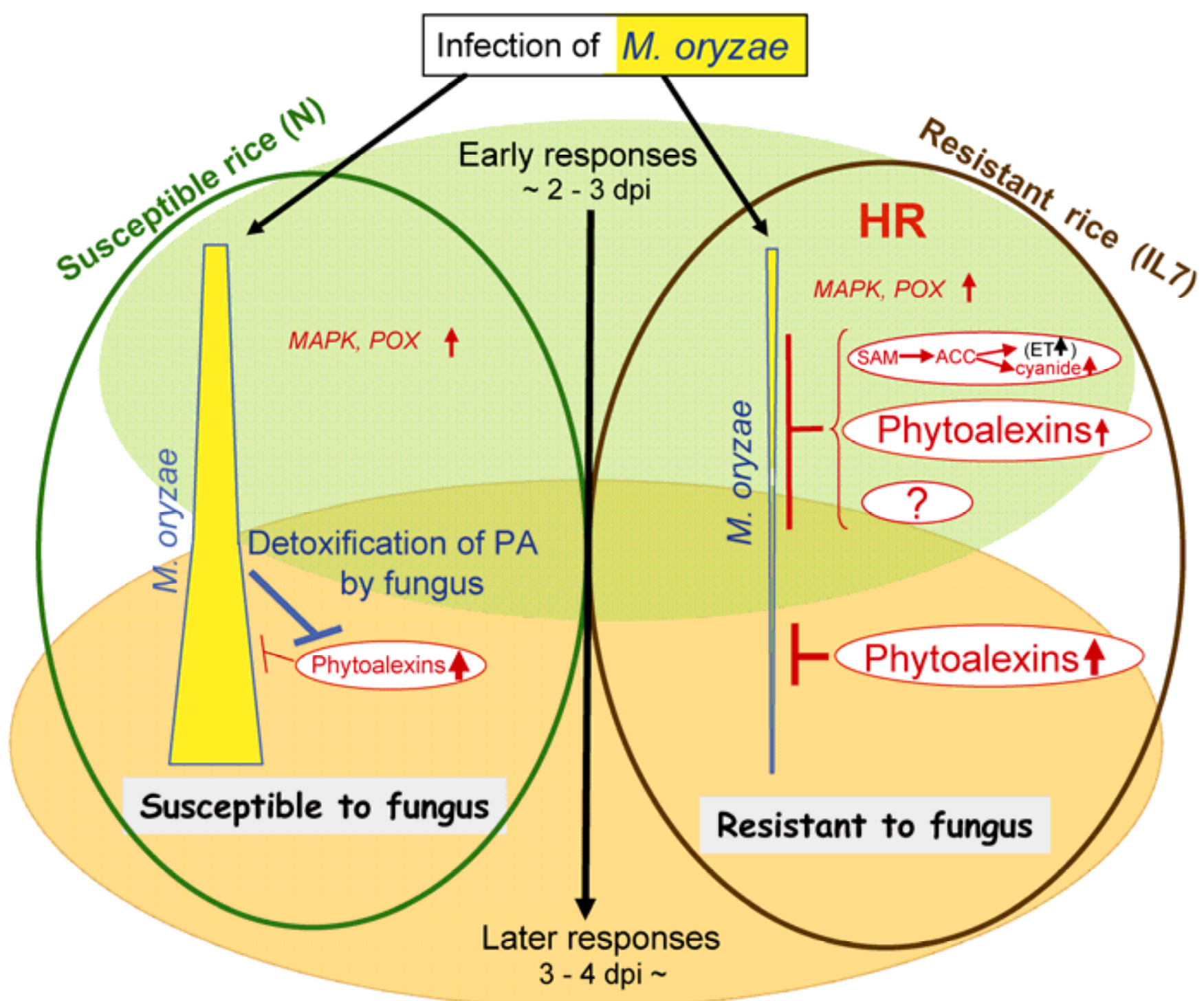

Fig. 8. Proposed role of phytoalexins (PA) on the resistance responses of rice to blast fungus. Predicted responses of resistant rice plants to blast fungus infection are compared with those in susceptible plants. The events occurring in the early (within 2 to 3 days postinoculation [dpi]) and later ( 3 to 4 dpi thereafter) responses are shown with green and orange backgrounds, respectively. Quantitative blast fungus growth in rice leaves is represented by the yellow areas with blue margins. Responses of the fungus are shown in blue and those of the host plant in red. In this system, expression of $M A P K$ and peroxidase $(P O X)$ genes is induced $1 \mathrm{~h}$ after inoculation in both Nipponbare and IL7 as the possible basal resistant response (Kuroda et al. 2006). In IL7 but not in Nipponbare, hypersensitive response (HR)-accompanied ethylene-cyanide biosynthesis, which may confer blast resistance, is induced in the early infection stage (Iwai et al. 2006). Furthermore, HR-accompanied accumulation of diterpene PA is enhanced with transient gene expression for PA synthetic genes, and the high levels of PA accumulated inhibit further fungal growth in resistant rice. On the other hand, in susceptible Nipponbare without HR, the fungus can grow in the early infection stage at which only low levels of PA are accumulated. In later stages, fungal growth continues accelerating detoxification of PA by the fungus. 


\section{Quantitative real-time \\ reverse transcription (RT)-PCR analysis.}

Total RNA was extracted from leaves using TRIzol reagent (Invitrogen, Carlsbad, CA, U.S.A.) in accordance with the manufacturer's instructions. First-strand cDNA was synthesized using an iScript cDNA synthesis kit (Bio-Rad, Hercules, CA, U.S.A.). Quantitative real-time RT-PCR was conducted using iQ SYBR green supermix (Bio-Rad) and an iCycler (Bio-Rad) in accordance with the manufacturer's instructions, with some modifications (Mitsuhara et al. 2008). At least three independent biological samples were used with gene-specific primers (Table 1). The data were normalized by the values of a rice actin transcript (AK071450), and relative transcript levels are shown with standard errors (SE).

\section{Analysis of momilactone A bioconversion by the suspension-cultured fungus.}

Fungal layers on PDA medium were cut into squares of approximately $1 \mathrm{~mm}^{2}$. The small pieces were suspended in PDA liquid medium and were incubated at $23^{\circ} \mathrm{C}$ in the dark with shaking at $120 \mathrm{rpm}$. After 4 or 5 days, $1 \mathrm{ml}$ of the fungal culture was put in a tube with a cap, and momilactone A was added to a final concentration of $0.3 \mathrm{mM}$. After incubation for appropriate time intervals, methanol was added to each sample to give a final concentration of $70 \%$. The PA and metabolites were extracted by gentle boiling in water for $5 \mathrm{~min}$. The filtrate was evaporated in vacuo, and the residue was recovered by $1 \mathrm{ml}$ of PDA medium for GC-MS analysis. When the PA in fungus and that in the medium were separately determined, 1 $\mathrm{ml}$ of the fungal culture was filtrated. Methanol was added to the residue precipitate and the filtrate to give a final concentration of $70 \%$. After heat treatment as mentioned above, these samples were filtrated, and the filtrates were evaporated to dryness in vacuo. The residue was recovered by $1 \mathrm{ml}$ of PDA liquid medium for GC-MS analysis and for germ-tube growth inhibition tests.

For GC-MS analysis, the aqueous solution was partitioned against ethyl acetate $(0.1 \mathrm{ml}$, three times). The combined ethyl acetate layer was evaporated to dryness in vacuo. The residue was dissolved in $10 \mu \mathrm{l}$ of acetone, and $1 \mu \mathrm{l}$ of the solution was injected into the GC-MS instrument (GCmate II; JEOL, Tokyo). Separation was carried out with a J\&W Scientific HP-5 column $(0.32 \mathrm{~mm}$ i.d. $\times 30 \mathrm{~m}, 0.25 \mu \mathrm{m}$ film thickness; Agilent Technologies, Santa Clara, CA, U.S.A.) under the following conditions: injector temperature, $280^{\circ} \mathrm{C}$; carrier gas, helium; flow rate, $1.0 \mathrm{ml} / \mathrm{min}$. The temperature program of a column oven was set to begin at $70^{\circ} \mathrm{C}$ for $1 \mathrm{~min}$, was elevated $10^{\circ} \mathrm{C}$ $\mathrm{min}^{-1}$ to $300^{\circ} \mathrm{C}$, and was maintained at $300^{\circ} \mathrm{C}$ for $3 \mathrm{~min}$. The conditions used for the mass spectrometer were as follows: ionization mode, EI $(70 \mathrm{eV})$; ion source temperature, $200^{\circ} \mathrm{C}$; scan range, $\mathrm{m} / \mathrm{z} 55$ to $700 ;$ scan rate, 1 s per scan.

\section{ACKNOWLEDGMENTS}

We thank T. Imbe and I. Ando of the National Agriculture and Food Research Organization for providing IL7 rice seeds, N. Hayashi of the National Institute of Agrobiological Sciences for providing M. oryzae race 003 and T. Iwai of Miyagi University for coaching the inoculation method. We also thank M. Teruse, T. Kimoto, and Y. Goto for their technical assistance. This study was partly supported by a grant from the Program for Promotion of Basic and Applied Researches for Innovations in Bio-oriented Industry (BRAIN).

\section{LITERATURE CITED}

Cartwright, D., Langcake, P., Pryce, R. J., Leworthy, D. P., and Ride, J. P. 1977. Chemical activation of host defense mechanisms as a basis for crop protection. Nature 267:511-513.
De Laat, A. M. M., and van Loon, L. C. 1983. The relationship between stimulated ethylene production and symptom expression in virus-infected tobacco leaves. Physiol. Plant Pathol. 22:261-273.

Dillon, V. M., Overton, L., Grayer, R. J., and Harborne, J. E. 1997. Differences in phytoalexin response among rice cultivars of different resistance to blast. Phytochemistry 44:599-603.

Grayer, R. J., and Kokubun, T. 2001. Plant-fungal interactions: The search for phytoalexins and other antifungal compounds from higher plants. Phytochemistry 56:253-263.

Gupta, A., and Chattoo, B. B. 2008. Functional analysis of a novel ABC transporter ABC4 from Magnaprthe grisea. FEMS (Fed. Eur. Microbiol. Soc.) Microbiol. Lett. 278:22-28.

Ise, K., and Horisue, N. 1988. Characteristics of several near-isogenic lines of rice for blast resistance gene. Breed Sci. (Suppl. In Japanese) 38:404405.

Iwai, T., Miyasaka, A., Seo, S., and Ohashi, Y. 2006. Contribution of ethylene biosynthesis for resistance to blast fungus infection in young rice plants. Plant Physiol. 142:1202-1215.

Kanno, Y., Otomo, K., Kenmoku, H., Mitsuhashi, W., Yamane, H., Oikawa, H., Toshima, H., Matsuoka, M., Sassa, T., and Toyomasu, T. 2006. Characterization of a rice gene family encoding type-A diterpene cyclases. Biosci. Biotechnol. Biochem. 70:1702-1710.

Kodama, O., Li W. X., Tamogami, S., and Akatsuka, T. 1992a. Oryzalexin $\mathrm{S}$, a novel stemarane-type diterpene rice phytoalexin. Biosci. Biotechol. Biochem. 56:1002-1003.

Kodama, O., Miyakawa, J., Akatsuka, T., and Kiyosawa, S. 1992b. Sakuranetin, a flavanone phytoalexin from ultraviolet-irradiated rice leaves. Phytochemistry 31:3807-3809.

Koga, J., Shimura, M., Oshima, K., Ogawa, N., Yamauchi, T., and Ogasawara, N. 1995. Phytocassanes A, B, C and D, novel diterpene phytoalexins from rice, Oryza sativa L. Tetrahedron 51:7907-7918

Koga, J., Ogawa, N., Yamauchi, T., Kikuchi, M., Ogasawara, N., and Shimura, M. 1997. Functional moiety for the antifungal activity of phytocassane E, a diterpene phytoalexin from rice. Phytochemistry 44:249253.

Kuć, J. 1995. Phytoalexins, stress metabolism, and disease resistance in plants. Annu. Rev. Phytopathol. 33:275-297.

Kuroda, K., Sasaki, K., Iwai, T., Yazaki Y., Hiraga, S., Seo, S., Mitsuhara, I., Minami, E., and Ohashi, Y. 2006. Rapid defense gene expression in both resistant and susceptible rice cultivars by elicitor(s) originating from conidia of blast fungus-Basal resistance response before fungal penetration into host cell. Physiol. Mol. Plant Pathol. 69:13-25.

Malamy, J., Carr, J. P., Klessig, D. F., and Laskin, I. 1990. Salicylic acid: A likely endogenous signal in the resistance response of tobacco to viral infection. Science 250:1002-1004.

Mayama S., Matsuura Y, Iida, H., and Tani, T. 1982. The role of avenalumin in the resistance of oat to crown rust, Puccinia coronata $\mathrm{f}$. $\mathrm{sp}$. avenae. Physiol. Plant Pathol. 20:189-199.

Mitsuhara I., Iwai, T., Seo, S., Yanagawa, Y., Kawahigasi, H, Hirose, S., Ohkawa, Y, and Ohashi, Y. 2008. Characteristic expression of twelve rice $\mathrm{PR} 1$ family genes in response to pathogen infection, wounding, and defense-related signal compounds. Mol. Genet. Genomics 279:415-427.

Müller, K. O., and Börger, H. 1940. Experimentelle Untersuchungen über die Phytophthora: Resistenz der Kartoffel. Arb. Biol. Reichsanst. Land Forstwirtsch. 23:189-231.

Pedras, M. S. C., and Ahiahonu P. W. K. 2002. Probing the phytopathogenic stem rot fungus with phytoalexins and analogues: Unprecedented glucosylation of camalexin and 6-methoxycamalexin. Bioorg. Med. Chem. 10:3307-3312.

Pedras, M. S. C., and Ahiahonu P. W. K. 2005. Metabolism and detoxification of phytoalexins and analogs by phytopathogenic fungi. Phytochemistry 66:391-411.

Pedras, M. S. C., and Khan, A. Q. 1997. Unprecedented detoxification of the cruciferous phytoalexin camalexin by a root phytopathogen. Bioorg. Med. Chem. Lett. 7:2255-2260.

Qi, M., and Young, Y. 2002. Quantification of Magnaporthe grisea during infection of rice plants using real-time polymerase chain reaction and northern blot/phosphoimaging analyses. Phytopathology 92:870-876.

Sasaki, K., Iwai, T., Hiraga, S., Kuroda, K., Seo, S., Mitsuhara, I., Miyasaka, A., Iwano, M., Ito, H., Matsui, H., and Ohashi, Y. 2004. Ten rice peroxidase redundantly respond to multiple stresses including infection with rice blast fungus. Plant Cell Physiol. 45:1442-1452.

Schuhegger, R., Rauhut, T., and Glawischnig, E. 2007. Regulatory variability of camalexin biosynthesis. J. Plant Physiol. 164:636-644.

Seo, S., Okamoto, M., Seto, H., Ishizuka, K., Sano, H., and Ohashi, Y. 1995. Tobacco MAP kinase: A possible mediator in wound signal transduction pathways. Science 270:1988-1992.

Seo, S., Seto, H., Yamakawa, H., and Ohashi, Y. 2001. Transient accumulation of jasmonic acid during the synchronized hypersensitive cell death 
in tobacco mosaic virus-infected tobacco leaves. Mol. Plant-Microbe Interact. 14:261-264.

Shimizu, T., Jikumaru, Y., Okada, A., Okada, K., Koga, J., Umemura, K. Minami, E., Shibuya, N., Hasegawa, M., Kodama, O., Nojiri, H., and Yamane, H. 2008. Effects of a bile acid elicitor, cholic acid, on the biosynthesis of diterpenoid phytoalexins in suspension-cultured rice cells. Phytochemistry 69:973-981

Snyder, B. A., and Nicholson, R. L. 1990. Synthesis of phytoalexins in sorghum as a site-specific response to fungal ingress. Science 248:1637-1639.

Stefanato, F. C., Abou-Mansour, E., Buchala, A., Kretschmer, M., Mosbach, A., Hahn, M., Bochet, C. G., Métraux, J.-P., and Schoonbeek, H.-J. 2009. The ABC transporter BcatrB from Botrytis cinerea exports camalexin and is a virulence factor on Arabidopsis thaliana. Plant J. 58:499-510.

Sun, C. B., Suresh, A., Deng, Y. Z., and Naqvi, N. I. 2006. A multidrug resistance transporter in Magnaporthe is required for host penetration and for survival during oxidative stress. Plant Cell 18:3686-3705.

Tomiyama, K. 1980. Resistance of potato against Phytophthora infestans, with a special reference to mechanisms of hypersensitive reaction. Plant Protection 34:2-8.
Toyomasu, T. 2008. Recent advances regarding diterpene cyclase genes in higher plants and fungi. Biosci. Biotechnol. Biochem. 72:1168-1175.

Umemura K., Ogawa, N., Shimura M., Koga J., Usami H., and Kono T. 2003. Possible role of phytocassane, rice phytoalexin, in disease resistance of rice against the blast fungus Magnaporthe grisea. Biosci. Biotechnol. Biochem. 67:899-902.

Urban, M., Bhargava, T., and Hamer, J. E. 1999. An ATP-driven efflux pump is a novel pathogenicity factor in rice blast disease. EMBO (Eur. Mol. Biol. Organ.) J. 18:512-521.

VanEtten, H. D., Pueppke, S. K., and Kelsey, T. C. 1975. 3,6a-Dihydroxy8,9-methylenedioxy pterocarpan as a metabolite of pisatin produced by Fusarium solani f. sp. pisi. Phytochemistry 14:1103-1105.

Xu, M., Wilderman, P. R., Morrone, D., Xu, J., Roy, A., Margis-Pinheiro, M., Upadhyaya, N. M., Coates, R. M., and Peters, R. J. 2007. Functional characterization of the rice kaurene synthase-like gene family. Phytochemistry 68:312-326.

Yamada, M., Kiyosawa, S., Yamaguchi, T., Hirano, T., Kobayashi, T., Kushibuchi, K., and Watanabe, S. 1976. Proposal of a new method for differentiating races of Pyricularia oryzae Cavara in Japan. Ann. Phytopath. Soc. Japan 42:216-219. 